\title{
An advanced in situ imaging method using heavy metal-doped hollow tubes to evaluate the biokinetics of carbon nanotubes in vivo
}

\author{
Shinsuke Kobayashi ${ }^{1}$, Shuji Tsuruoka ${ }^{2}$, Yuki Usui ${ }^{3}$, Hisao Haniu ${ }^{4}$, Kaoru Aoki ${ }^{1}$, Seiji Takanashi ${ }^{1}$, \\ Masanori Okamoto ${ }^{1}$, Hiroki Nomura ${ }^{1}$, Manabu Tanaka ${ }^{1}$, Shigetoshi Aiso ${ }^{5}$, Misae Saito ${ }^{5}$, Hiroyuki Kato ${ }^{1}$ and \\ Naoto Saito ${ }^{4}$
}

The biosafety of carbon nanotubes (CNTs) is a concern, and CNT biokinetics is a key biosafety issue. CNTs labeled with carbon isotopes, functionalized with moieties, or coated with colloidal metal particles have been used to monitor CNT biokinetics, though major technical issues (such as isotope preparation and handling or change in CNT surface properties) remain. The present report aims at establishing an advanced and simple in situ imaging method of CNTs monitoring in vivo involving the use of CNTs filled with heavy metal particles (peapods). Gadolinium (Gd)-peapods containing $\mathrm{GdCl}_{3}$ were synthesized using doublewalled CNTs. The limits of Gd-peapods detection on magnetic resonance imaging (MRI) in solutions and agarose gel cubes were $1.3 \mu \mathrm{g} \mathrm{ml}^{-1}$ and $4 \mu \mathrm{g} \mathrm{ml}^{-1}$, respectively. The peapods in rats was easily visualized by MRI and the change in signal intensity was dose dependent. This newly developed method can be used to monitor CNT biokinetics in vivo without tedious tissue preparation.

NPG Asia Materials (2015) 7, e203; doi:10.1038/am.2015.77; published online 17 July 2015

\section{INTRODUCTION}

Industrial application of carbon nanotubes (CNTs) as highperformance composites, conductive additives in secondary batteries and corrosion-preventing coatings is increasing. ${ }^{1}$ The possibility that CNTs cause serious lesions such as mesotheliomas because they are nano fibrous materials similar in shape to asbestos is a concern. Takagi et $a .^{2}$ and Poland et al. ${ }^{3}$ reported that peritoneal administration of multi-walled CNTs induce inflammation and mesothelioma-like lesions in abdomen mesothelium. Prior to those reports, CNTs were used in rigorous biological evaluations. ${ }^{4,5}$ Mercer et al. ${ }^{6}$ showed that multi-walled CNT distribution to the lymph nodes, diaphragm, chest wall and extrapulmonary organs in mice after inhalation exposure, indicating the need to investigate CNTs biokinetics. In addition to occupational exposures, exposure through biomaterial, medical diagnosis and treatment applications is a concern. ${ }^{7}$ Particularly in drug-delivery systems and imaging applications, CNTs are directly injected in veins and their whereabouts in vivo must be essentially understood. Unavoidably, CNTs used as scaffolds for tissue regeneration are transferred to the other organs through the blood and lymphatic circulation. Thus, methods of CNT biokinetics evaluation are needed urgently.
CNT biokinetics has been investigated after intravenous administration..$^{8-18}$ One method of in vivo CNT distribution monitoring is to assay tissue preparations of all organs after CNTs are administered. However, preparations vary and measurement is not quantitative. ${ }^{9}$ Although single-walled CNTs can be assayed in vivo by Raman spectroscopy owing to its intrinsic signatures, multi-walled CNTs including double-walled CNTs (DWCNTs) cannot be assayed using this method. ${ }^{10}$ Many current methods propose labeling CNTs with carbon isotopes of ${ }^{13} \mathrm{C}$ or ${ }^{14} \mathrm{C},{ }^{8,12,18}$ and functionalizing the surface with carboxyl groups or coat the surface with colloidal metal particles. ${ }^{14-17}$ Labeling requires a special facility to handle and synthesize isotope-labeled CNTs. Preparing labeled CNTs is not difficult but the physical, chemical or biological characteristics of the original CNTs may be modified in the process. ${ }^{19,20}$ Furthermore, exfoliation of functionalized moieties or surface substances will occur during measurement. ${ }^{21}$ Therefore, an alternative method that does not require tissue preparation would be desirable.

The present work aims at development of an advanced in situ imaging method of CNT kinetics evaluation in vivo. DWCNTs filled with heavy metal particles were prepared to achieve our goal. Doped CNTs (called peapods) are designated doped particles@CNT types. As such, peapod technology is expected in the future to find a broad

\footnotetext{
${ }^{1}$ Department of Orthopaedic Surgery, Shinshu University School of Medicine, Matsumoto, Japan; ${ }^{2}$ Aquatic Innovation Center, Shinshu University, Nagano, Japan; ${ }^{3}$ Aizawa Hospital Sports Medicine Center, Matsumoto, Japan; ${ }^{4}$ Institute for Biomedical Sciences, Interdisciplinary Cluster for Cutting Edge Research, Shinshu University, Matsumoto, Japan and ${ }^{5}$ Japan Bioassay Research Center, Japan Industrial Safety and Health Association, Hadano, Japan

Correspondence: Professor N Saito, Institute for Biomedical Sciences, Interdisciplinary Cluster for Cutting Edge Research, Shinshu University, Asahi 3-1-1, Matsumoto 390-8621, Japan.
} 
range of in vivo applications as nanocontainers. ${ }^{22}$ The first peapod, $\mathrm{C}_{60} @$ single-walled CNT, was synthesized using fullerene $\mathrm{C}_{60} \cdot{ }^{23,24}$ However, $\mathrm{C}_{60}$ modifications significantly alter CNT characteristics. In the present work, marker molecules were directly inserted into the cylindrical hollow structure unique to CNTs. ${ }^{25-27}$ The peapods were used to evaluate biokinetics in tissues and in vivo. We found that the distribution of CNTs in the body can be determined without tissue preparation.

\section{MATERIALS AND METHODS \\ Peapods}

DWCNTs used in this study were obtained from Toray Industries (Tokyo, Japan). The average diameter, average length and density were $2 \mathrm{~nm}, 2 \mu \mathrm{m}$ and $1.83 \mathrm{~g} \mathrm{ml}^{-1}$, respectively. Density was estimated from transmission electron microscopy (TEM) images (JEM-2100 equipped with Cs-corrected unit EM-Z07167T, JOEL, Tokyo, Japan). The average diameter and length were provided by Toray. Supplied DWCNTs were washed in deionized pure water and ethanol to remove dispersant and residues, and then heated at $383 \mathrm{~K}$ to remove moisture. Gadolinium (Gd)-peapods were synthesized using a glassware apparatus depicted in Supplementary Figure S1. In all, $100 \mathrm{mg}$ of DWCNTs were weighed on an electronic balance and placed in the main tube of a two-way Pyrex glass tube. One hundred milligrams of $\mathrm{GdCl}_{3} \cdot 6 \mathrm{H}_{2} \mathrm{O}$ (Wako Pure Chemical Industries, Osaka, Japan) was placed in a side tube of the apparatus. The main tube was heated in a heating mantle at $423 \mathrm{~K}$ and degassed using a vacuum pump. This degassing was conducted in two steps; the DWCNTs were first dried and then a target pressure was achieved. After reaching $10^{-5} \mathrm{~Pa}$ inside the tube, the tube neck was melted and sealed. The main and side tubes were placed in a heating mantle at $773 \mathrm{~K}$ for $24 \mathrm{~h}$, cooled down to room temperature and peapods were taken out from the main tube. The Gd-peapods were washed in weak acid and then ethanol, rinsed in pure water, dried at $373 \mathrm{~K}$ in air overnight and characterized using TEM and X-ray fluorescence (XRF) analysis (XRF ZSX Primus II, Rigaku, Tokyo, Japan). The $\mathrm{X}$-ray attenuation coefficient of the DWCNTs was measured in an XRD system (Ultima IV, Rigaku). The mass attenuation coefficients of $\mathrm{Pb}, \mathrm{Gd}, \mathrm{Cl}$ and $\mathrm{Al}$ were obtained from the literature. ${ }^{28}$ The magnetic properties of peapods were evaluated using a vibrating sample magnetometer (PM-VSM-191483-MRO, Tamagawa, Saitama, Japan). After testing an empty acrylic sample container for its magnetic properties, the same container with a previously weighed peapod sample $(0.019 \mathrm{~g})$ was re-tested using the same procedures. The magnetic contribution of the container was subtracted to obtain the magnetic properties of the peapods.

\section{MRI determination of detection limit of Gd-peapods}

Brightness ratio and detection limit of Gd in saline solution were determined using MRI. A 1:10000 dilution in saline of Gadolisome (DS Pharma Biomedical, Osaka, Japan), a Gd solution $\left(0.9 \mathrm{mg} \mathrm{ml}^{-1} \mathrm{Gd}\right)$ used for small animal testing, and a saline solution (the control) were placed in $0.6-\mathrm{ml}$ microtubes which were inserted into an MRI scanner (MRmini SA (1.5 T), Neomax Engineering, Gunma, Japan). The parameters of 2D-SE and T1-intensified acquisition were: 500/9 (ratio of repetition time (TR) to echo time), $90^{\circ}$ ( $0 \mathrm{~dB}$; flip angle (FA)), $20 \mathrm{~mm}$ (field of view) and $2 \mathrm{~mm}$ (slice thickness). Axial images of microtubes were acquired and their brightness was determined at 4, 8 and $12 \mathrm{~mm}$ from the tip of the tube. Signal intensity was calculated as an average of signal intensities. The same procedure was repeated with Gd-peapods dispersed in $20 \mathrm{ml}$ of aqueous solution including $0.1 \mathrm{wt} \%$ Tween 80 (NOF, Tokyo, Japan; polyoxyethylene sorbitan monooleate) as dispersant and sonicated for $40 \mathrm{~min}$ at room temperature. This solution was diluted 100-1000-fold, placed in 0.6-ml microtubes and evaluated as above in MRI images.

\section{Agarose cube making and MRI optimization}

To optimize the quality of MRI images of organs, agarose gel was used as a surrogate organ prior to animal tests. Gd-peapods were suspended in saline solution at $500 \mu \mathrm{g} \mathrm{ml}^{-1}$ including $0.1 \mathrm{wt} \%$ Tween 80 , sonicated for $30 \mathrm{~min}$, mixed with $1 \mathrm{wt} \%$ agarose gel (Takara Bio, Shiga, Japan) and shaped into a cube $(1 \mathrm{ml})$. Cubes containing $1-250 \mu \mathrm{g} \mathrm{ml}^{-1} \mathrm{Gd}$-peapods were prepared. Also control cubes were made with saline only. The $250-\mu \mathrm{g} \mathrm{ml}^{-1}$ Gd-peapod cube and the control cube were placed side-by-side in the MRI scanner and signal intensities were measured to determine optimal TR and FA. The other machine parameters were fixed: 3D-Flash, field of view $40 \mathrm{~mm} \phi$, double accumulation and slice thickness $1.25 \mathrm{~mm}$. TR was adjusted at 10, 30, 50, 70 and $100 \mathrm{~ms}$, and contrast-to-noise ratio (CNR) was calculated using the results of inter-tissues method (air noise). Then, CNR values at $0-10 \mathrm{~dB}$ were measured after correcting for MRI attenuation and an optimal CNR was determined.

\section{In vivo test with rats}

Solutions for rat tail injection included Gd-peapods dispersed in saline with $0.1 \mathrm{wt} \%$ Tween 80 and diluted to concentrations of 25, 125 and $250 \mu \mathrm{g} \mathrm{ml}^{-1}$ Gd-peapods, pristine CNTs dispersed in saline with $0.1 \mathrm{wt} \%$ Tween 80 and diluted to 25,125 and $250 \mu \mathrm{g} \mathrm{ml}^{-1}$ CNT solutions and saline with Tween 80 (the control). Seven-week-old male Wistar rats (Japan SLC, Shizuoka, Japan) were anesthetized using an anesthesia apparatus for small animals (Narcobit, Natsume Seisakusho, Tokyo, Japan) and injected into the tail vein with the preparations after antisepticization with ethanol. No rat death occurred post exposure. Five rats per group were killed at $1 \mathrm{~h}, 24 \mathrm{~h}, 1$ week and 3 weeks post exposure. An incision was made and internal organs were surgically removed to expose the thoracic cavity and trachea. During the operation, $6 \mathrm{ml}$ of $20 \%$ neutral buffered formaldehyde was injected through the trachea into the lung through a 21-G needle to prevent lung collapse. The lungs were removed after the trachea was tied, to avoid escape of the formaldehyde solution, and before the liver, spleen and kidneys were removed. Those removed organs were fixed in the formaldehyde solution overnight.

Contrast in organ samples was measured on MRI images acquired under the optimized conditions determined above, with saline in a microtube placed into the MRI sensing chamber and serving as a brightness control. CNR was calculated from those MRI pictures. After MRI signal analysis, the organs were histopathologically evaluated. Left and right lungs were cut along the major bronchi, embedded in paraffin, sliced, stained with hematoxylin and eosin, and observed under optical and polarized light microscopes. The other organs were prepared and evaluated as above. CNR values were statistically analyzed with SPSS Statistics (version 21.0, IBM, NY, USA). A generalized linear model was adopted using time and concentration as fixed parameters.

All animal experimentation procedures were carried out in compliance with the guidelines of the institutional animal care committee of Shinshu University.

\section{RESULTS}

\section{Synthesis and characterization of DWCNTs and peapods}

Figures 1a and b show a TEM image and XRF analysis of Gd-peapods, respectively. The hollow tubular space of DWCNTs is obviously filled with $\mathrm{GdCl}_{3}$ particles. It is difficult to determine the volume of the hollow space occupied by particles because definitive particle size and density cannot be determined from this analysis. However, the particle volume estimated from the TEM image is $<10 \%$ of that of DWCNTs. As $\mathrm{GdCl}_{3}$ particles are within the tubes, and the particle density and nominal DWCNTs density are $\sim 4500 \mathrm{~kg} \mathrm{~m}^{-3}$ and $1830 \mathrm{~kg} \mathrm{~m}^{-3}$, respectively, the mass percentage of $\mathrm{GdCl}_{3}$ in the Gd-peapod does not exceed 3 even if the particles were to fully occupy the DWCNT inner tubes. Mass percentage of $\mathrm{Gd}$ particles in peapods was 2.1 atom $\%$ by XRF and simple analysis. In the TEM picture, most of the $\mathrm{GdCl}_{3}$ particles were inside the hollow space, even though $\mathrm{GdCl}_{3}$ was predicted to adhere to the outer DWCNT surface. This finding suggests that the $\mathrm{GdCl}_{3}$ on the Gd-peapod surface was washed away by the present procedure. As a result, the interior of DWCNTs was mostly filled by $\mathrm{GdCl}_{3}$ particles. To confirm that $\mathrm{Gd}$-peapods were well synthesized, the X-ray attenuation coefficient was measured as shown in Figure 1c. Although the mass attenuation coefficient of Gd-peapods is not as large as that of lead, the mass of Gd is large enough to attenuate X-rays (unlike the masses of chloride and 
a
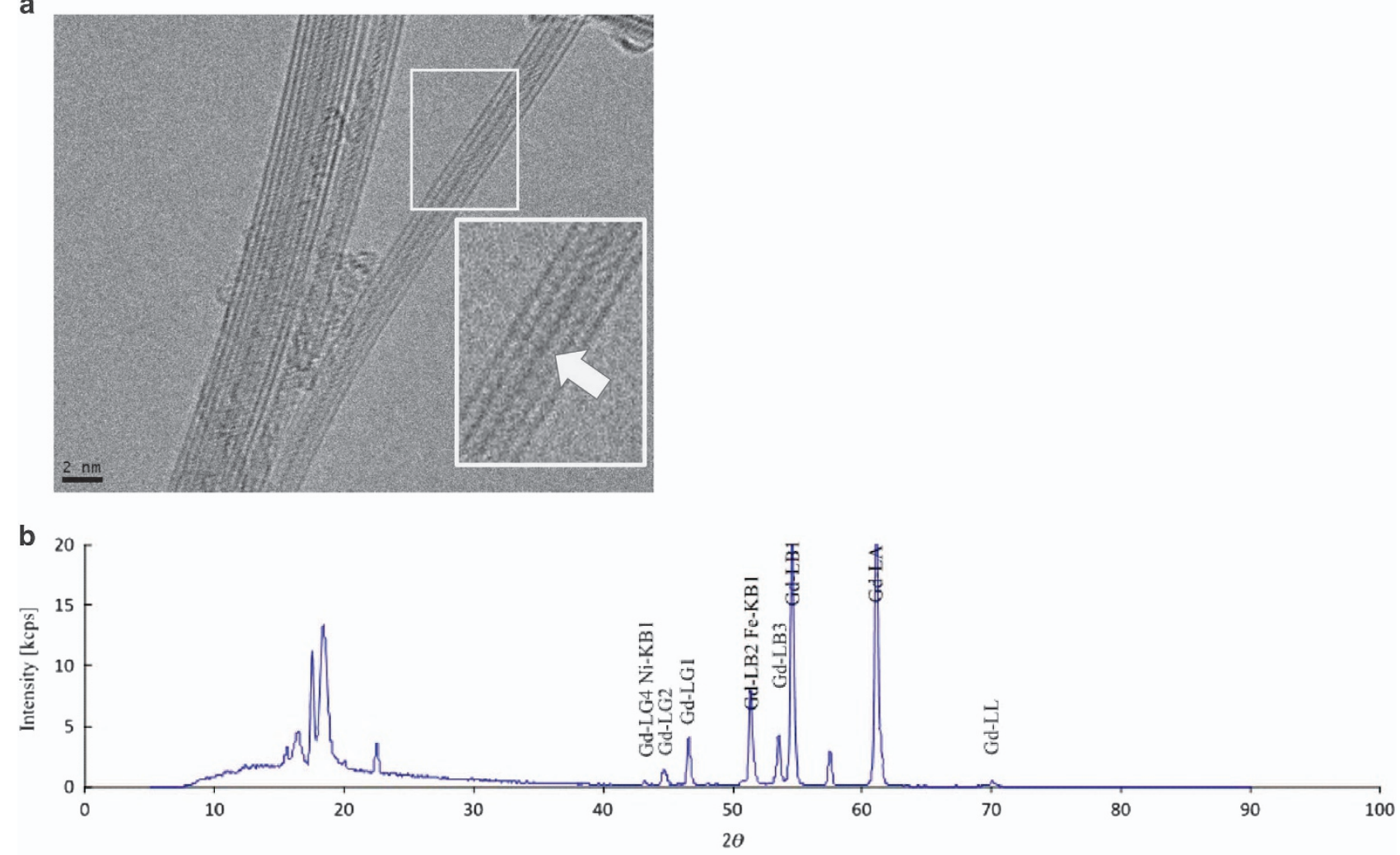

C

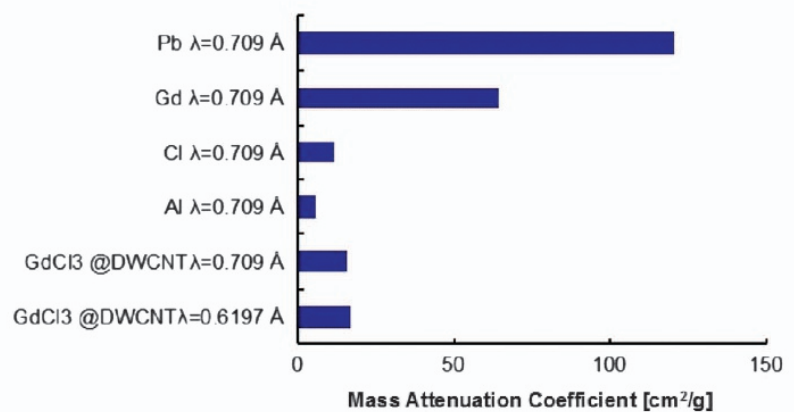

d

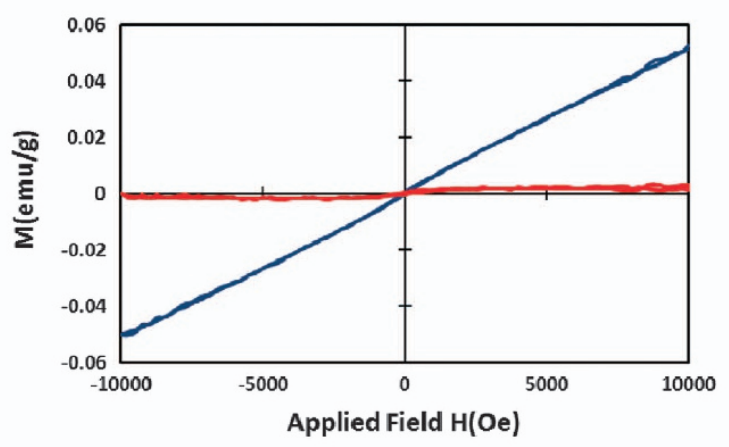

—Gd-Peapod —Pristine CNTs

Figure 1 Characterization of gadolinium (Gd)-peapods. (a) A transmission electron microscopy (TEM) picture of Gd-peapods. GdCl 3 particles fill the hollow part of the inner carbon nanotube (white arrow). (b) X-ray fluorescence (XRF) analysis spectrum of Gd-peapods. The mass ratio of carbon and Gd were 91.3770 and 2.1016 mass percentage, respectively. (c) Mass attenuation coefficient of Gd-peapods. (d) Magnetic properties of Gd-peapods and empty peapods. Only Gd-peapods exhibit paramagnetic characteristics with a positive magnetic susceptibility. 

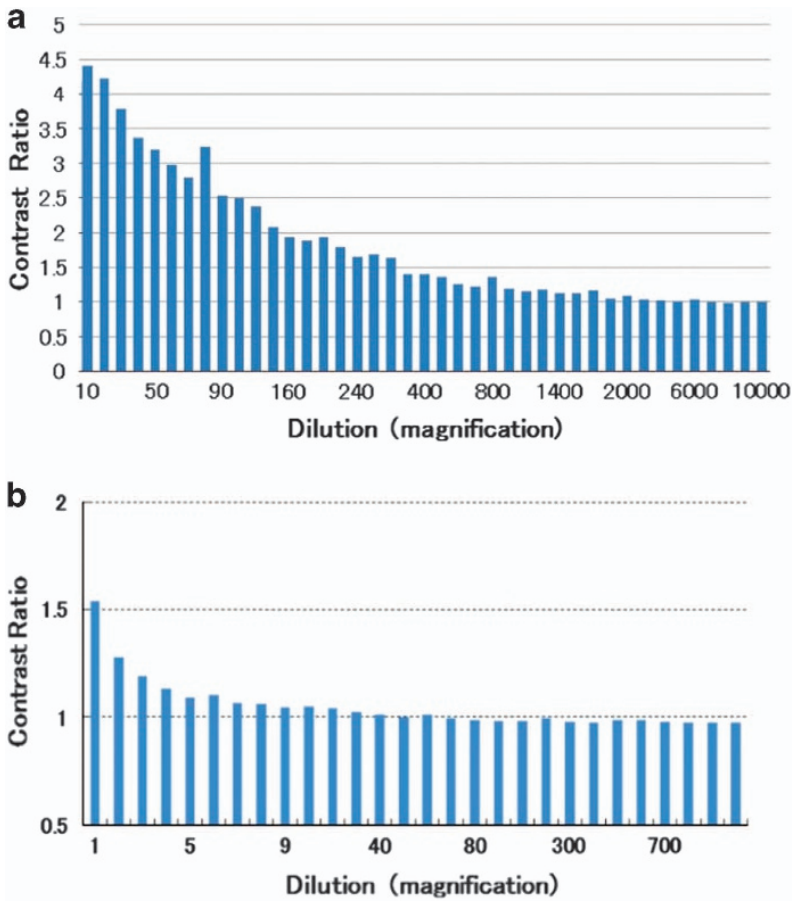

Figure 2 Relationship of contrast to magnification in magnetic resonance imaging (MRI) used to find limits of gadolinium (Gd) contrast media and Gd-peapods detection. (a) Detection limit of Gd contrast medium. The contrast ratio of $\mathrm{Gd}$ contrast medium remained constant after diluting $\geqslant 7000$ times ( $\mathrm{Gd}$ concentration: $130 \mathrm{ng} \mathrm{m}^{-1}$ ). (b) Detection limit of Gd-peapods. The contrast ratio of Gd-peapods and Gd in saline after diluting $\geqslant 50$ times (Gd-peapods concentration: $1.3 \mu \mathrm{g} \mathrm{ml}^{-1}$, Gd concentration: $40 \mathrm{ng} \mathrm{ml}^{-1}$ ). aluminum). Therefore, the Gd-peapod was well synthesized in the present study. An evaluation of Gd-peapod magnetic properties showed a positive magnetic susceptibility of $\sim 5.24 \mathrm{E}-6 \mathrm{emu} \mathrm{g}{ }^{-1}$ Oe. The data obtained from the curve for the magnetic susceptibility of simple-substance CNTs were linear and almost zero; therefore, the Gd-peapods were thought to encapsulate a paramagnetic substance (Figure 1d).

Determination of detection limits in MRI with Gd contrast media Prior to Gd-peapod MRI, a detection limit of Gd was determined by referencing to known concentrations of Gadolisome. Contrast ratio of Gadolisome diluted $\geqslant 7000$ times was equal to that of saline only, indicating that the lower threshold of Gd detection on MRI was $130 \mathrm{ng} \mathrm{ml}^{-1}$ (Figure 2a). Likewise, the lower threshold of Gd detection in Gd-peapods on MRI was $1.3 \mu \mathrm{g} \mathrm{ml}^{-1}$ of Gd-peapods or $40 \mathrm{ng} \mathrm{ml}^{-1}$ of Gd (Figure 2b). The limit of detection was lower in Gd-peapods than in Gd solution, probably because the structure of Gd-peapods $\left(\mathrm{GdCl}_{3}\right.$ particles surrounded by graphene sheet rolls, forming a continuous lining within hollow tubes) is unique.

\section{MRI determination of a Gd detection limit in agarose gel filled with Gd-peapods}

Gd-peapods were embedded in agarose gel (used as a model system or surrogate organ) and measured on MRI to determine a Gd detection limit. One-ml agarose gel cubes containing saline with or without Gd-peapods were compared. CNR was obtained from the signal intensity of those cubes using the inter-tissues method (air noise). TR and FA of peapod cubes were compared with those of control cubes. Figure 3a shows two 3D-flash exposures: $5000 \mathrm{~ms}$ in echo time, $1.25 \mathrm{~mm}$ of slice width (32 slices) and constant field of view at $40 \mathrm{~mm}$ ø. A CNR peak appears at $50 \mathrm{~ms}$ after a TR change. With a
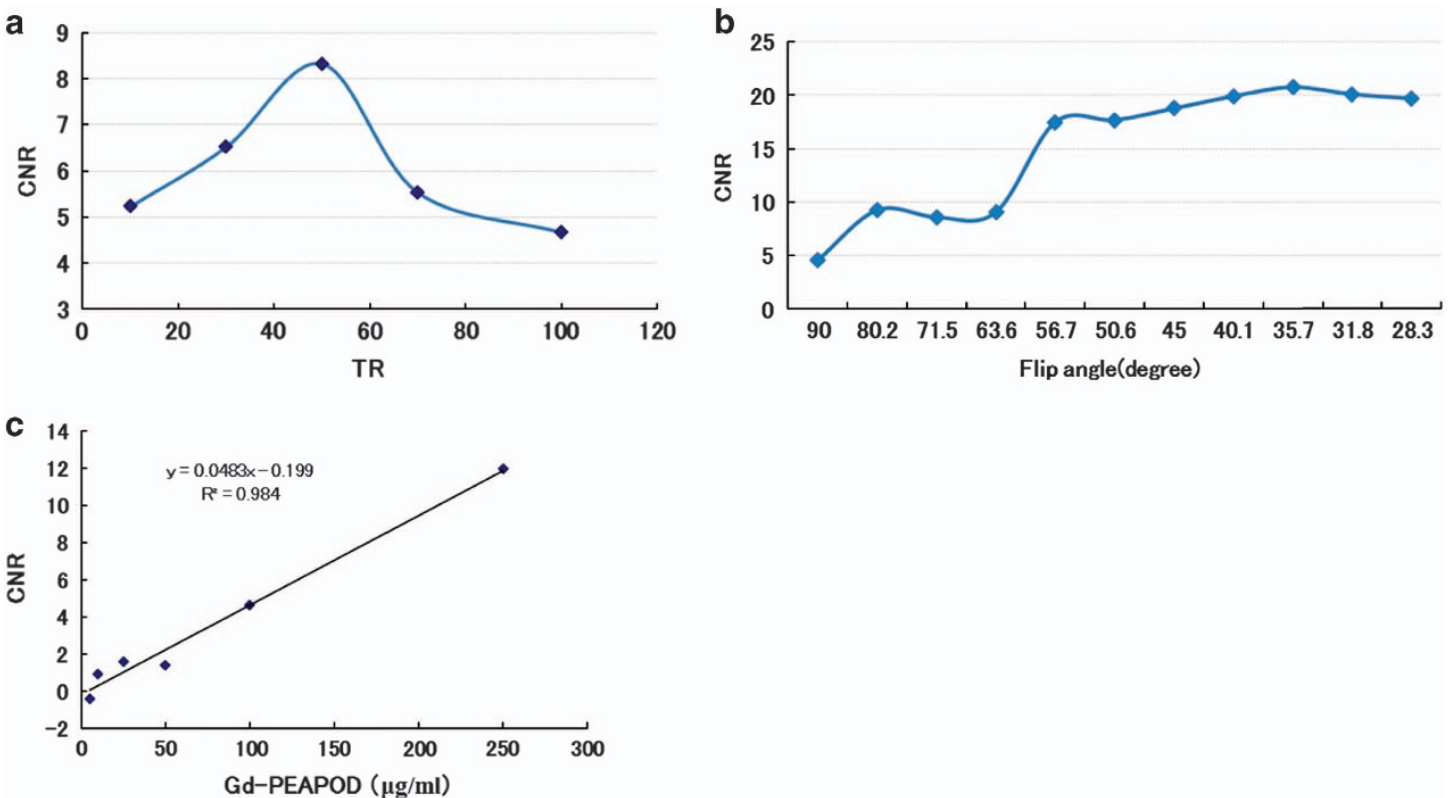

Figure 3 Magnetic resonance imaging (MRI) detection in agarose gel filled with Gd-peapods. (a) Contrast-to-noise ratio (CNR) of agarose gel with Gd-peapods by repetition time (TR) adjustment. A CNR peak appears with a TR of $50 \mathrm{~ms}$. (b) CNR of agarose gel with Gd-peapods by flip angle (FA) adjustment. As FA decreases, the CNR increases and peaks at $35.7^{\circ}$. However, FA decreases at $\leqslant 35.7^{\circ}$, and resolution of the images is reduced. (c) An evaluation of gel cube filled with Gd-peapods using MRI. The CNR depends on Gd-peapods concentration. Using a calibration curve, we determined that the detection limit was $4 \mu \mathrm{g} \mathrm{m} l^{-1}$. 
change of FA, CNR starts increasing at $90^{\circ}$ and reaches a plateau at $35.7^{\circ}$ (Figure $3 \mathrm{~b}$ ). Notably, FA was fixed at $90^{\circ}$ in routine measurements. With an FA change, CNR did not increase at $\leqslant 35.7^{\circ}$, and contrast resolution decreased at the four corners of the image. Thus, the optimal TR and FA were $50 \mathrm{~ms}$ and $35.7^{\circ}$, respectively. Likewise, the limit of Gd detection in agarose gel cubes containing Gd-peapods was determined using a CNR calibration curve over a range of
Gd-peapod concentrations. The detection limit was $4 \mu \mathrm{g} \mathrm{ml}^{-1}$ of Gd-peapods, at which the CNR reaches zero (Figure 3c).

MR imaging of removed organs after tail vein injection of Gd-peapods

Gd-peapods (concentrations: 250, 125 and $25 \mu \mathrm{g} \mathrm{ml}^{-1}$ ) and the original CNTs (concentration: $250 \mu \mathrm{g} \mathrm{ml}^{-1}$ ) in saline were a

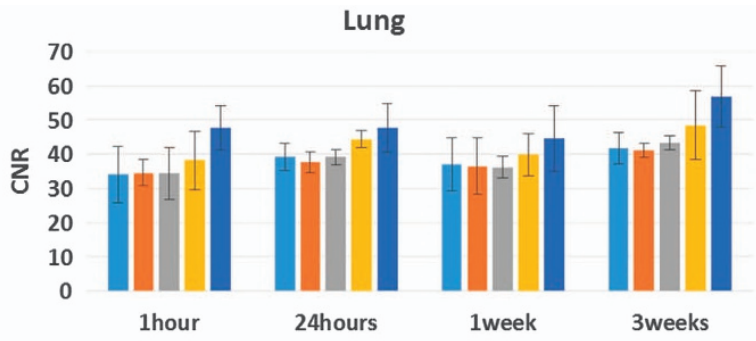

Spleen

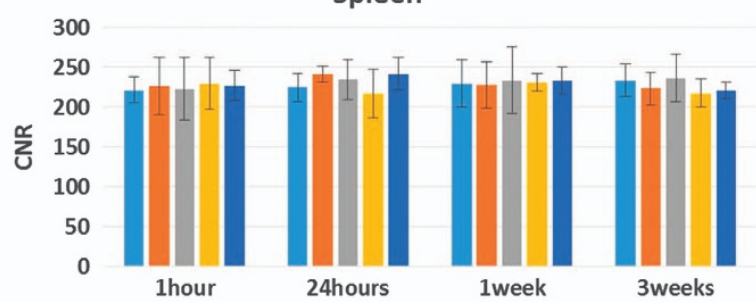

200

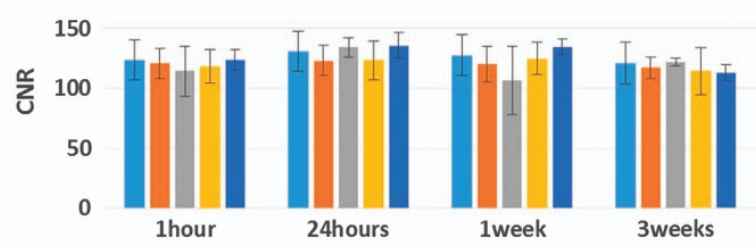

Kidney

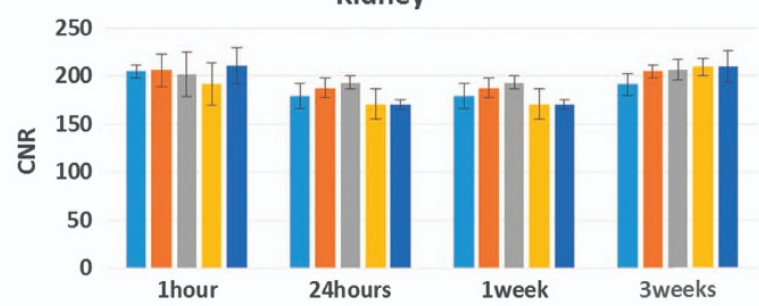

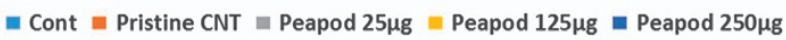

b

$$
\begin{aligned}
& \text { pristine } \\
& \text { DWCNT }
\end{aligned}
$$

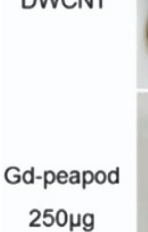
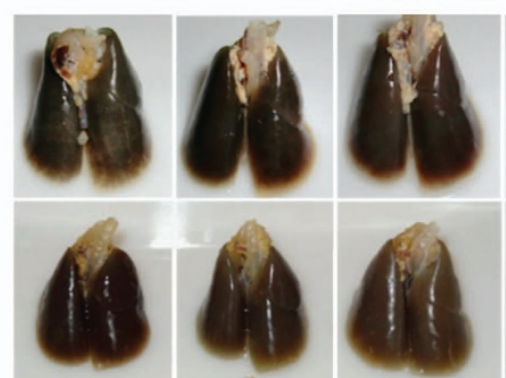

Gd-peapod $125 \mu \mathrm{g}$

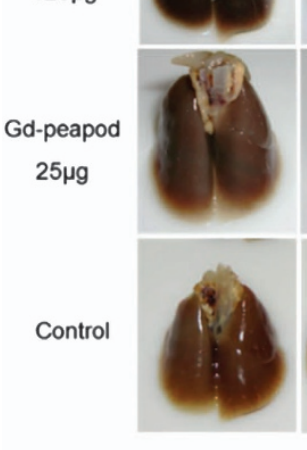

1 hour

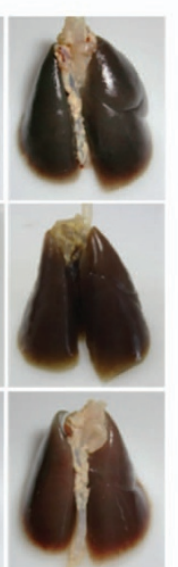

C

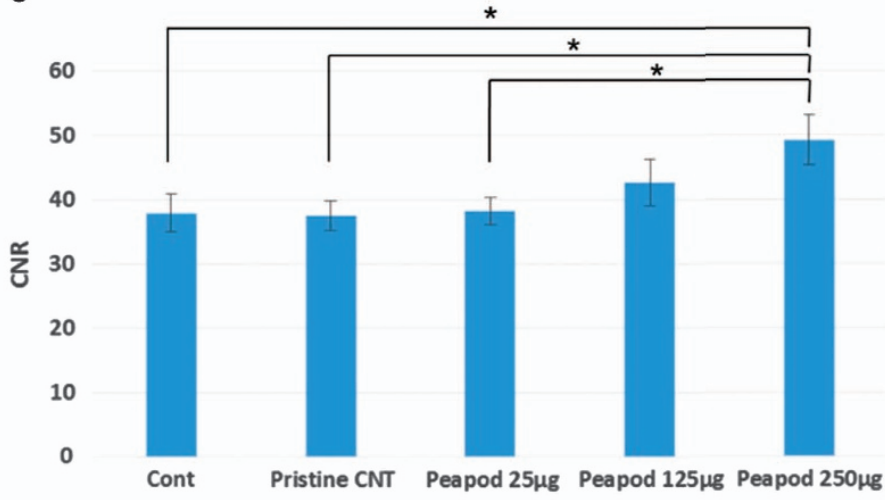

Figure 4 Magnetic resonance imaging (MRI) and macroscopic evaluation after tail vein injection of Gd-peapods. (a) The contrast-to-noise ratio (CNR) after injection. The CNR value in lungs increases with peapod concentration. However, individual samples do not show a significant time-dependence. The CNR values in livers, spleens, or kidneys from rats injected with Gd-peapods do not increase. (b) A matrix showing color changes in lungs. Time after the injection (vertical) versus peapod concentration (horizontal). Even after 3 weeks, lungs injected with pristine carbon nanotubes (CNTs) and Gd-peapods remain black. (c) CNR value in lungs change with peapod concentration change. The highest Gd-peapod concentration (250 $\mu$ g) results in significant increase in CNR, in contrast to the control, pristine CNTs and Gd-peapods $\left(25 \mu \mathrm{g} ;{ }^{*} P<0.05\right)$. 
a

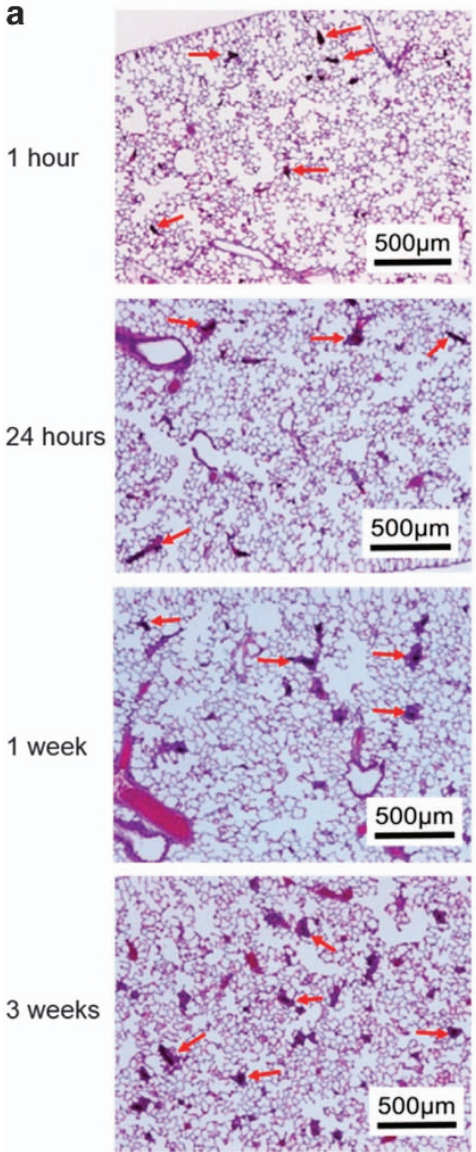

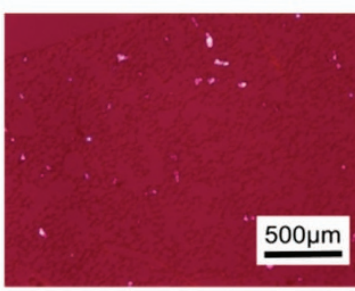

b
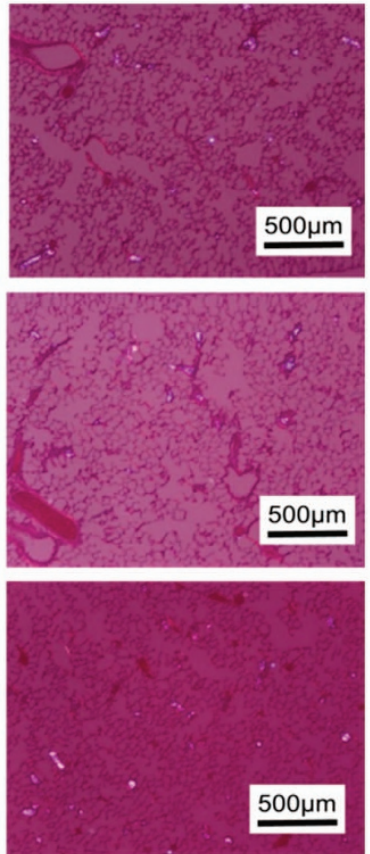

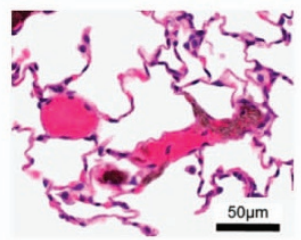

1hour

24hours
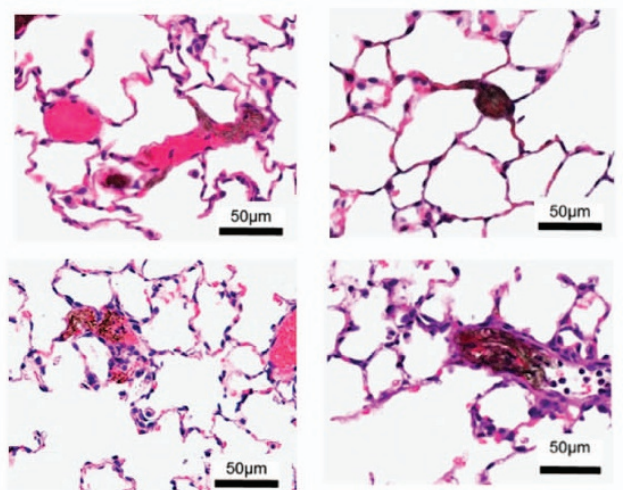

1week

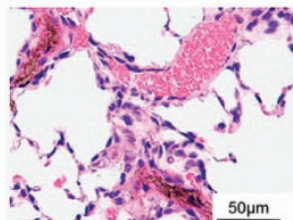

3weeks

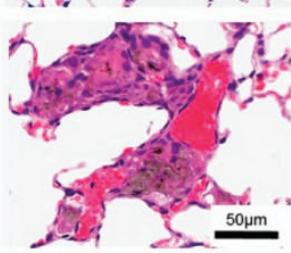

Pristine CNT

$(250 \mu \mathrm{g} / \mathrm{ml})$
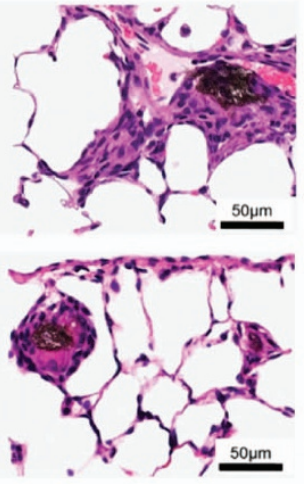

Gd-Peapod

$(250 \mu \mathrm{g} / \mathrm{ml})$

Figure 5 Histological evaluations of the lungs after tail vein injection of Gd-peapods. Red arrows indicate individual carbon nanotube (CNT) aggregates. (a) Optical microscopic views showing the histology of the lungs removed $1 \mathrm{~h}, 24 \mathrm{~h}, 1$ week and 3 weeks after injection. CNTs deposition can be observed. White particles with high luminance can be observed under a polarizing microscope (right line). (b) Optical microscope images of removed lungs at high magnification. At $1 \mathrm{~h}$ and $24 \mathrm{~h}$ after injection, aggregates of Gd-peapods and the pristine CNTs extend from the pulmonary artery to peripheral capillaries, however, none are observed in the pulmonary vein. At 1 week and 3 weeks after injection, small granulomas have formed around aggregates of Gd-peapods and pristine CNTs. Macrophages are observed inside some of the pulmonary alveoli. These findings were similar in both the pristine CNT and peapod groups.

administered via rat tail vein injection $(n=20)$. Tween 80 was used as dispersant in all samples. Saline with the dispersant was also administered as a control. Five rats per group were killed at $1 \mathrm{~h}$, $24 \mathrm{~h}, 1$ week and 3 weeks after the administration. Their lungs, liver, spleen and kidneys were removed surgically and imaged on MRI to measure CNR values. The CNR value in lungs increased in a dosedependent manner as Gd-peapod concentration increased, without statistical significance in individual samples. On the other hand, the CNR values in the other organs from rats treated with Gd-peapods did not increase (Figure $4 \mathrm{a}$ ). Figure $4 \mathrm{~b}$ shows that tail vein injection of Gd-peapods turned all lungs black. Figure 4c shows the lung CNRs of individual groups $(n=20)$. The lung CNR value was significantly higher in the $250 \mu \mathrm{g} \mathrm{ml}^{-1}$ peapod group than the control group $(P=0.001)$, pristine CNT group $(P=0.002)$ and the $25 \mu \mathrm{g} \mathrm{ml}^{-1}$ peapod group $(P=0.006)$. However, no significant difference was observed between the $250 \mu \mathrm{g} \mathrm{ml}^{-1}$ and $125 \mu \mathrm{g} \mathrm{ml}^{-1}$ peapod groups.

\section{Histological evaluations of organs removed from rats administered} Gd-peapods

After the MRI signal intensity measurement, those organs removed from the Gd-peapod and control groups were stained with hematoxylin and eosin and observed under optical and polarizing microscopes in order to evaluate time-dependent changes histopathologically. Liver, spleen and kidney samples remained unchanged. Lungs with Gd-peapods showed carbon deposition (Figure 5a). The peapods were agglomerated in blood vessels, particularly in the pulmonary artery (extending to peripheral capillaries), but not in the pulmonary vein. Moreover, granulomas were present around these agglomerations at 1 and 3 weeks after exposure to Gd-peapods and pristine CNTs (Figure 5b). No intense neutrophil inflammatory response was present in any sample. There was no difference between the Gd-peapod and pristine CNT histological findings.

\section{DISCUSSION}

The advantage of the present MRI method is that image diagnosis can be used to biokinetically evaluate CNTs, and peapods can be synthesized using CNTs without drastically changing the surface morphology. Specifically, the surfaces of Gd-peapods synthesized by leaving CNTs and $\mathrm{GdCl}_{3}$ in a heated vacuum chamber for a while are not significantly altered. Thus, a method with Gd-peapods is feasible for determining distribution and concentration of CNTs in the living body without a major modification of the original CNT bioactivity. The proposed in situ method is a new approach to evaluate CNTs biokinetics in vivo. TEM shows that surface morphology of peapods (CNTs filled with target particles that do not change bioactivity significantly) are identical to CNTs without target particles. 
Taking quantum mechanical effects into consideration, we conclude that doping CNTs with particles does not affect their surface characteristics markedly. ${ }^{29,30}$ One can see the 'necklace chains' of $\mathrm{GdCl}_{3}$ within each CNT, indicating that CNTs can be measured quantitatively at specific locations on MRI. CNTs usually blend into the background. Highly pure CNTs were reported to exhibit diamagnetism with a negative magnetic susceptibility of approximately $-2 \mathrm{E}-6 \mathrm{emug}^{-1} \mathrm{Oe}$. In the present study as well, almost no magnetic properties were observed. On the contrary theGd-peapods used in the present study were found to have a magnetic susceptibility of approximately $5.24 \mathrm{E}-6 \mathrm{emu} \mathrm{g}^{-1} \mathrm{Oe}$. The paramagnetism resulting from filling CNTs with gadolinium is a characteristic suitable for their application as a tracing material in vivo. ${ }^{31}$

To establish an in situ method of biokinetics evaluation, detection limits were demonstrated on MRI analysis. The results indicate that our approach is promising. Tail vein administration of Gd-peapods resulted in significant differences in CNR between peapods in lungs and peapods in saline solution or between peapods in saline and pristine CNTs in saline solution; there was no difference in CNR between pristine CNTs and saline, though the lung color was very different from that of the control. It suggests that the accumulation and persistence of peapods in the lungs intensified MRI signals significantly. Histopathologically, Gd-peapod aggregates were found in the pulmonary artery and capillaries in all parts of the lungs, which corresponded to the increase found in MRI signal intensity, but no aggregates were found in the livers, spleens and kidneys and no increase in MRI signal intensity was found in those organs. The change in signal intensity was dose-dependent in all samples so that this protocol is applicable to quantitative determination of peapod biokinetics.

As far as we know, no studies have evaluated the biokinetics of gadolinium-encapsulated peapods as in the present study, although one study evaluated the biokinetics of CNTs with gadoliniummodified surfaces. ${ }^{32}$ Judging from the mass ratio of $\mathrm{Gd}$ to $\mathrm{Cl}$ atoms by XRF analysis, the substance within DWCNTs was identified as $\mathrm{GdCl}_{3}$ (Figure $1 \mathrm{~b}$ ). Elemental mapping analysis by energy dispersive $\mathrm{X}$-ray spectroscopy revealed both $\mathrm{Gd}$ and $\mathrm{Cl}$ in $\mathrm{CNT}$ bundles, with $\mathrm{GdCl}_{3}$ encapsulated in the CNTs and not found outside (data not shown). With regard to the amount of gadolinium encapsulated, no quantitative method is currently available for measuring concentrations within the peapod interior except XRF. Essentially XRF analysis is a nondestructive technique, so it allows direct measurement of the amount of gadolinium encapsulated in peapods used for animal experimentation, but in case of inductive coupled plasma atomic emission spectrometry or another destructive analytical method Gdpeapods sample will be completely destroyed. With this in mind, in the present study, we obtained adequate accuracy through relative evaluation of peapod concentration by determining the percentage mass ratio of Gd in peapods using XRF measurements, and comparing the results with known Gd concentrations measured on MRI images. To confirm Gd-peapod stability in saline, the amount of Gd in saline after leaving Gd-peapods in saline for 6 months was evaluated by MRI. The signal intensity of Gd-peapods remained high, with no significant desorption of Gd particles observed (Supplementary Figure S2). In addition, TEM examination revealed the absence of Gd leakage even after electron-beam irradiation. For these reasons, it can be concluded that Gd-peapods are also stable in vivo and do not release Gd.

Adjustment of MRI parameters is critical to in vivo measurement of peapods distribution. The FA was initially set to $90^{\circ}$ for Flash Mode photo-taking and to intensify the T1-weighted image. In the present work a detection limit was achieved with TR at $50 \mathrm{~ms}$ after MRI adjustment, which gave the best 3D-Flash images. With TR at $50 \mathrm{~ms}$, the highest CNT resolution was achieved with FA at $35.7^{\circ}$. However, this FA did not give good contrast (low CNR value) because the total signal intensity was low. Alternatively, conditions to obtain a higher CNR were sought. The signal contrast was improved with initial TR set at $50 \mathrm{~ms}$. The optimal FA was $35.7^{\circ}$. The higher FA increased the $\mathrm{CNR}$, but the CNR between tissues decreased. The parameter settings were, after all, judged optimal for measurement of peapods distribution in vivo.

The limits of Gd-peapods detection in solutions and agarose gel cubes were $1.3 \mu \mathrm{g} \mathrm{ml}^{-1}$ and $4 \mu \mathrm{g} \mathrm{ml}^{-1}$, respectively. Liu et al. ${ }^{10}$ reported a CNT (single-walled) detection limit of $0.02-4 \mu \mathrm{g} \mathrm{ml}^{-1}$ using Raman spectroscopy. These detection limits of CNT distribution measurement in the living body are the highest to be published so far. In the present study, the CNTs detection limits were similar, though it is not appropriate to compare both results because Liu et al. ${ }^{10}$ used a destructive method that cannot be used for multi-walled CNTs (including DWCNTs), whereas our method allows in situ measurement.

The proposed method is useful for in vivo investigation because it does not require sample slicing or incineration of tissues to weigh the carbon amount in tissues. This technique might be useful particularly in the application of drug-delivery systems and in imaging with the aim of seeing how CNTs circulate and accumulate in tissues and organs. In conclusion, the first in situ method suitable for CNTs biokinetics evaluation was developed and demonstrated.

\section{CONFLICT OF INTEREST}

The authors declare no conflict of interest.

\section{ACKNOWLEDGEMENTS}

This work is part of the research program 'Development of innovative methodology for safety assessment of industrial nanomaterials' supported by the Ministry of Economy, Trade and Industry (METI) of Japan.

Author contributions: SK, ShT and NS designed the study, analyzed the data and wrote the manuscript. YU, SK, KA, SeT, HN, MT, SA and MS performed in vivo experiments. SK, ShT, $\mathrm{HH}$ and $\mathrm{MO}$ performed in vitro experiments. $\mathrm{HK}$ and NS supervised the project. All authors discussed the results and helped with the preparation of the final manuscript.

1 Endo, M., Strano, M. S. \& Ajayan, P. M. Potential applications of carbon nanotubes. Top. Appl. Phys. 111, 13-61 (2008).

2 Takagi, A., Hirose, A., Nishimura, T., Fukumori, N., Ogata, A., Ohashi, N., Kitajima, S. \& Kanno, J. Induction of mesothelioma in $\mathrm{p} 53+/$ - mouse by intraperitoneal application of multi-wall carbon nanotube. J. Toxicol. Sci. 33, 105-116 (2008).

3 Poland, C. A., Duffin, R., Kinloch, I., Maynard, A., Wallace, W. A., Seaton, A., Stone, V., Brown, S., Macnee, W. \& Donaldson, K. Carbon nanotubes introduced into the abdominal cavity of mice show asbestos-like pathogenicity in a pilot study. Nat. Nanotechnol. 3, 423-428 (2008).

4 Royal Society and Royal Academy of Engineering Nanoscience and nanotechnologies: opportunities and uncertainties. RS Policy Document 19/04 (2004).

5 Oberdörster, G., Maynard, A., Donaldson, K., Castranova, V., Fitzpatrick, J., Ausman, K., Carter, J., Karn, B., Kreyling, W., Lai, D., Olin, S., Monteiro-Riviere, N., Warheit, D. \& Yang, H. ILSI Research Foundation/Risk Science Institute Nanomaterial Toxicity Screening Working Group. Principles for characterizing the potential human health effects from exposure to nanomaterials: elements of a screening strategy. Part. Fibre. Toxicol. 2, 8 (2005).

6 Mercer, R. R., Scabilloni, J. F., Hubbs, A. F., Wang, L., Battelli, L. A., McKinney, W., Castranova, V. \& Porter, D. W. Extrapulmonaru transport of MWCNT following inhalation exposure. Part. Fibre. Toxicol. 10, 38 (2013).

7 Saito, N., Haniu, H., Usui, Y., Aoki, K., Hara, K., Takanashi, S., Shimizu, M., Narita, N., Okamoto, M., Kobayashi, S., Nomura, H., Kato, H., Nishimura, N., Taruta, S. \& Endo, M. Safe clinical use of carbon nanotubes as innovative biomaterials. Chem. Rev. 114, 6040-6079 (2014). 
8 Deng, X., Jia, G., Wang, H., Sun, H. \& Wang, X. X. Translocation and fate of multi-walled carbon nanotubes in vivo. Carbon NY 45, 1419-1424 (2007).

9 Yang, S. T., Guo, W., Lin, Y., Deng, X. Y., Wang, H. F., Sun, H. F., Liu, Y. F., Wang, X. Wang, W., Chen, M., Huang, Y. P. \& Sun, Y. P. Biodistribution of pristine single-walled carbon nanotubes in vivo. J. Phys. Chem. C 111, 17761-17764 (2007).

10 Liu, Z., Davis, C., Cai, W., He, L., Chen, X. \& Dai, H. Circulation and long-term fate of functionalized, biocompatible single-walled carbon nanotubes in mice probed by Raman spectroscopy. Proc. Natl Acad. Sci. USA 105, 1410-1415 (2008).

11 Al Faraj, A., Cieslar, K., Lacroix, G., Gaillard, S., Canet-Soulas, E. \& Crémillieux, Y. A. In vivo imaging of carbon nanotube biodistribution using magnetic resonance imaging. Nano Lett. 9, 1023-1027 (2009).

12 Georgin, D., Czarny, B., Botquin, Magali., Mayne-L'Hermite, M., Pinault, M., Bouchet-Fabre, B., Carriere, M., Poncy, J. L., Chau, Quang., Maximilien, R., Dive, V. \& Taran, F. Preparation of 14C-labeled multiwalled carbon nanotubes for biodistribution investigations. J. Am. Chem. Soc. 131, 14658-14659 (2009).

13 Al Faraj, A., Fauvelle, F., Luciani, N., Lacroix, G., Levy, M., Crémillieux, Y. \& Canet-Soulas, E. In vivo biodistribution and biological impact of injected carbon nanotubes using magnetic resonance techniques. Int. J. Nanomed. 6, 351-361 (2011)

14 Wu, H., Liu, G., Zhuang, Y., Wu, D., Zhang, H., Yang, H., Hu, H. \& Yang, S. The behavior after intravenous injection in mice of multiwalled carbon nanotube / Fe304 hybrid MRI contrast agents. Biomaterials 32, 4867-4876 (2011).

15 Al-Jamal, K. T., Nunes, A., Methven, L., Ali-Boucetta, H., Li, S., Toma, F. M., Herrero, M. A., Al-Jamal, W. T., ten Eikelder, H. M., Foster, J., Mather, S., Prato, M., Bianco, A. \& Kostarelos, K. Degree of chemical functionalization of carbon nanotubes determines tissue distribution and excretion profile. Chem. Int. Ed. Engl. 51, 6389-6393 (2012).

16 Doan, B. T., Seguin, J., Breton, M., Le Beherec, R., Bessodes, M., Rodríguez-Manzo, J. A., Banhart, F., Beloeil, J. C., Scherman, D. \& Richard, C. Functionalized single-walled carbon nanotubes containing traces of iron as new negative MRI contrast agents for in vivo imaging. Contrast Media Mol. Imaging 7, 153-159 (2012).

17 Yin, M., Wang, M., Miao, F., Ji, Y., Tian, Z., Shen, H. \& Jia, N. Water-dispersible multiwalled carbon nanotube/iron oxide hybrids as contrast agents for cellular magnetic resonance imaging. Carbon NY 50, 2162-2070 (2012).

18 Czarny, B., Georgin, D., Berthon, F., Plastow, G., Pinault, M., Patriarche, G., Thuleau, A., L'Hermite, M. M., Taran, F. \& Dive, V. Carbon nanotube translocation to distant organs after pulmonary exposure: insights from in situ (14)C-radiolabeling and tissue radioimaging. ACS Nano 8, 5715-5724 (2014).

19 Tsuruoka, S., Takeuchi, K., Koyama, K., Noguchi, T., Endo, M., Tristan, F. S., Terrones, M., Matsumoto, H., Saito, N., Usui, Y., Porter, D. W. \& Castranova, V. ROS evaluation for a series of CNTs and their derivatives using an ESR method with DMPO. J. Phys. Conf. Ser. 429, 012029 (2013).

20 Singh, R., Pantarotto, D., Lacerda, L., Pastorin, G., Klumpp, C., Prato, M., Bianco, A. \& Kostarelos, K. Tissue biodistribution and blood clearance rates of intravenously administered carbon nanotube radiotracers. Proc. Natl Acad. Sci. USA 103 3357-3362 (2006)
21 Liu, X., Gurel, V., Morris, D., Murray, D. W., Zhitkovich, A., Kane, A. B. \& Hurt, R. H. Bioavailability of nickel in single-wall carbon nanotubes. Adv. Mater. 19 2790-2796 (2007).

22 Prato, M., Kostarelos, K. \& Bianco, A. Functionalized carbon nanotubes in drug design and discovery. Acc. Chem. Res. 41, 60-68 (2008).

23 Smith, B. W., Monthioux, M. \& Luzzi, D. E. Encapsulated C60 in carbon nanotubes. Nature 396, 323-324 (1998).

24 Krive, I. V., Shekhter, R. I. \& Jonson, M. Carbon "peapods"- a new tunable nanoscale graphitic structure (review). Low Temp. Phys. 32, 887-905 (2006).

25 Shimada, T., Okaszaki, T., Taniguchi, R., Sigai, T. \& Shinohara, H. Ambipolar field-effect transistor behavior of Gd@C[sub 82] metallofullerene peapods. Appl. Phys. Lett. 81, 4067-4069 (2002).

26 Kim, Y. A., Kojima, M., Muramatsu, H., Umemoto, S., Watanabe, T., Yoshida, K. Sato, K., Ikeda, T., Hayashi, T., Endo, M., Terrones, M. \& Dresselhaus, M. S. In situ raman study on single- and double-walled carbon nanotubes as a function of lithium insertion. Small 2, 667-676 (2006).

27 Fujimori, T., Batista dos Santos, R., Hayashi, T., Endo, M., Kaneko, K. \& Tománek, D. Formation and properties of selenium double-helices inside double-wall carbon nanotubes: experiment and theory. ACS Nano 7, 5607-5613 (2013).

28 Harold, P. K. \& Leroy, E. A. X-Ray Diffraction Procedures, second edition (John Wiley \& Sons, 1974).

29 Kaneko, K., Ishii, C., Ruike, M. \& Kuwabara, H. Origin of superhigh surface area and microcrystalline graphitic structures of activated carbons. Carbon NY 30, 1075-1088 (1992).

30 Kitaura, R. \& Shinohara, H. Endohedral metallofullerenes and nano-peapods. Jpn. J. Appl. Phys. 46, 881-891 (2007).

31 Lipert, K., Ritschel, M., Leonhardt, A., Krupskaya, Y., Buchner, B. \& Klingeler, R. Magnetic properties of carbon nanotubes with and without catalyst. J. Phys. Conf. Ser. 200, 072061 (2010).

32 Marangon, I., Ménard-Moyon, C., Kolosnjaj-Tabi, J., Béoutis, M. L., Lartigue, L., Alloyeau, D., Pach, E., Ballesteros, B., Autret, G., Ninjbadgar, T., Brougham, D. F., Bianco, A. \& Gazeau, F. Covalent functionalization of multi-walled carbon nanotubes with a gadolinium chelate for efficient T1-weighted magnetic resonance imaging. Adv Funct. Mater. 24, 7173-7186 (2014).

(i) This work is licensed under a Creative Commons Attribution 4.0 International License. The images or other third party material in this article are included in the article's Creative Commons license, unless indicated otherwise in the credit line; if the material is not included under the Creative Commons license, users will need to obtain permission from the license holder to reproduce the material. To view a copy of this license, visit http:// creativecommons.org/licenses/by/4.0/

Supplementary Information accompanies the paper on the NPG Asia Materials website (http://www.nature.com/am) 